\title{
Generate, Test, and Aggregate
}

\section{A Calculation-based Framework for Systematic Parallel Programming with MapReduce ${ }^{\star}$}

\author{
Kento Emoto ${ }^{1}$, Sebastian Fischer ${ }^{\star \star}$, and Zhenjiang $\mathrm{Hu}^{2}$ \\ 1 The University of Tokyo \\ 2 National Institute of Informatics, Tokyo
}

\begin{abstract}
MapReduce, being inspired by the map and reduce primitives available in many functional languages, is the de facto standard for large scale data-intensive parallel programming. Although it has succeeded in popularizing the use of the two primitives for hiding the details of parallel computation, little effort has been made to emphasize the programming methodology behind, which has been intensively studied in the functional programming and program calculation fields. We show that MapReduce can be equipped with a programming theory in calculational form. By integrating the generate-and-test programing paradigm and semirings for aggregation of results, we propose a novel parallel programming framework for MapReduce. The framework consists of two important calculation theorems: the shortcut fusion theorem of semiring homomorphisms bridges the gap between specifications and efficient implementations, and the filter-embedding theorem helps to develop parallel programs in a systematic and incremental way. We give nontrivial examples that demonstrate how to apply our framework.
\end{abstract}

\section{Introduction}

MapReduce [6], the de facto standard for large scale data-intensive applications, is a remarkable parallel programming model, allowing for easy parallelization of data intensive computations over many machines in a cloud. It is used routinely at companies such as Yahoo!, Google, Amazon, and Facebook. Despite its abstract interface that effectively hides the details of parallelization, data distribution, load balancing, and fault tolerance, developing efficient MapReduce parallel programs remains as a challenge in practice.

As a concrete example, consider the known statistics problem of inferring a sequence of hidden states of a probabilistic model that most likely causes a sequence of observations [13] (see details in Section 6). This problem is important

\footnotetext{
* An extended version of this paper including more explanations and an additional section on generalizing our approach to arbitrary algebraic data types is available as Technical Report METR2011-34, University of Tokyo, http://www.keisu.t.utokyo.ac.jp/research/techrep/

$\star \star$ supported by the German Academic Exchange Service (DAAD) 
in natural language processing and error code correction, but it is far from easy for one to come up with an efficient MapReduce program to solve it. The problem becomes more difficult, if we would like to find the most likely sequence with additional requirements such that the sequence should contain a specific state exactly five times, or that the sequence should not contain a specific state anywhere after another. The main difficulty in programming with MapReduce is that nontrivial problems are usually not in a simple divide-and-conquer form that can be easily mapped to MapReduce without producing an exponential number of intermediate candidates. Moreover, the inputs may not just form a simple set of elements as in MapReduce; rather they are often structured as lists.

The MapReduce framework was inspired by the map and reduce (fold) primitives available in many functional languages. Although it has successfully popularized the use of these two primitives for hiding the details of parallel computation, little effort has been made to emphasize the programming methodology behind, which has been intensively studied in functional programming and program calculation [1, 3, 8, 14, 22]. This lack of programming methodology for MapReduce has led to publication of too many papers about MapReduce applications [18], each addressing a solution to one specific problem, even if quite a lot of problems follow a common pattern and can be solved generally.

To remedy this situation, we will show that MapReduce can be equipped with a programming theory in calculational form [3, 15, 24, which can be applied to give efficient solutions to a wide class of problems. For illustration, we consider a general class of problems which can be specified in the following generate-testand-aggregate (GTA for short) form (here, o denotes function composition):

\section{aggregate o test $\circ$ generate}

Problems that match this specification can be solved by first generating possible solution candidates, then keeping those candidates that pass a test of a certain condition, and finally selecting a valid solution or making a summary of valid solutions with an aggregating computation. For example, the above statistics problem may be informally specified by generating all possible sequences of state transitions, keeping those that satisfy a certain condition, and selecting one that maximizes the products of probabilities (see Section 6).

Like other programming theories in calculational form [15, 24], the big challenges in the development of our calculation theory are to decide a structured form such that any program in this form is guaranteed to be efficiently parallelized, and to show how a specification can be systematically mapped to the structured form. To this end, we refine the specification with constraints on each of its components.

- The generator should be parallelizable in a divide-and-conquer manner and polymorphic over semiring structures, guaranteeing that the final program can be coded with MapReduce efficiently.

- The condition for the test should be defined structurally in terms of a list homomorphism. 
- The aggregator should be a semiring computation (semiring homomorphism), guaranteeing that the aggregating computation is structured in a way that matches with the generator.

These constraints, as will be seen later, can be satisfied for practical problems such as the statistics problem mentioned above. An interesting result of this paper is that any specification that satisfies these constraints can be automatically mapped to an efficient parallel program in, but not limited to, MapReduce.

In this paper, by integrating the generate-and-test programing paradigm and semirings for result aggregation, we propose a novel parallel programming framework that is centered on two calculation theorems, the semiring fusion theorem and the filter embedding theorem. These two calculation theorems play an important role for the systematic development of efficient parallel programs in MapReduce for a problem that is specified by a semiring-polymorphic generator, a test with a homomorphic predicate, and a semiring homomorphism as aggregator. Our main technical contributions can be summarized as follows.

- We propose a new formalization of GTA problems in the context of parallel computation based on the semiring fusion theorem. We show how a generator can be specified as a list homomorphism polymorphic over semirings, an aggregator can be specified as a semiring homomorphism, and fusion of their composition can be done for free and results in an efficient homomorphism parallelizable by MapReduce.

- We propose a new systematic and incremental development of parallel programs for more complicated GTA problems based on the filter embedding theorem. The filter-embedding theorem allows a semiring homomorphism to absorb preceding tests yielding a new semiring homomorphism. We give nontrivial examples that demonstrate how to apply our framework.

The rest of the paper is organized as follows. We start with background on lists, monoids, homomorphisms, and MapReduce in Section 2 Then, after exemplifying our approach to specify parallel programs by means of the knapsack problem in Section 3, we focus on two important calculation theorems, the shortcut fusion theorem for semiring homomorphisms in Section 4, and the filter embedding theorem in Section [5. We discuss a more complex application in Section 6] Finally, we discuss related work in Section 7 and conclude in Section 8

\section{Background: Lists, Monoids and MapReduce}

The notation in this paper is reminiscent of Haskell [2]. Function application is denoted by a space and the argument may be written without brackets, so that $(f a)$ means $f(a)$ in ordinary notation. Functions are curried: they always take one argument and return a function or a value, and the function application associates to the left and binds more strongly than any other operator, so that $f a b$ means $(f a) b$ and $f a \otimes b$ means $(f a) \otimes b$. Function composition is denoted by $\circ$, and $(f \circ g) x=f(g x)$ according to its definition. Binary operators can be used as functions by sectioning as follows: $a \oplus b=(a \oplus) b=(\oplus b) a=(\oplus) a b$. 


\subsection{Lists, Monoids, and Homomorphisms}

Lists are finite sequences of values of the same type. A list is either empty, a singleton, or the concatenation of two other lists. We write [] for the empty list, $[x]$ for the singleton list with element $x$, and $x s+y s$ for the concatenation of two lists $x s$ and ys. For example, the term [1] + [2] \# [3] denotes a list with three elements, often abbreviated as $[1,2,3]$. We write $[A]$ for the type of lists with elements of type $A$.

Definition 1 (Monoid). Given a set $M$ and a binary operator $\odot$ on $M$, the pair $(M, \odot)$ is called a monoid if and only if $\odot$ is associative and has an identity $\imath_{\odot} \in M$ :

$$
\begin{aligned}
& (a \odot b) \odot c=a \odot(b \odot c) \\
& \imath \odot \odot a=a=a \odot \imath \odot
\end{aligned}
$$

For example, $([A],+)$ is a monoid: $\#$ is associative and [] is its identity.

Homomorphisms are structure preserving mappings. In the case of monoids they respect the binary operation and its identity.

Definition 2 (Monoid Homomorphism). Given two monoids $(M, \odot)$ and $\left(M^{\prime}, \odot^{\prime}\right)$, a function hom: $M \rightarrow M^{\prime}$ is called monoid homomorphism from $(M, \odot)$ to $\left(M^{\prime}, \odot^{\prime}\right)$ if and only if:

$$
\begin{aligned}
\text { hom } \imath \odot & =\imath \odot^{\prime} \\
\text { hom }(x \odot y) & =\text { hom } x \odot^{\prime} \text { hom } y
\end{aligned}
$$

For example, the function sum for summing up all elements in a list is a monoid homomorphism from $([\mathbb{Z}],+)$ to $(\mathbb{Z},+)$ :

$$
\begin{array}{ll}
\operatorname{sum}[] & =0 \\
\operatorname{sum}[x] & =x \\
\operatorname{sum}(x s+y s) & =\text { sum } x s+\text { sum ys }
\end{array}
$$

There is more than one monoid homomorphism from $([\mathbb{Z}],+)$ to $(\mathbb{Z},+)$ but the property sum $[x]=x$ characterizes sum uniquely, because $[A]$ is the free monoid over $A$ : for every result monoid, a list homomorphism (monoid homomorphism from lists) is characterized uniquely by its result on singleton lists.

List homomorphisms are relevant to parallel programming because associativity allows to distribute the computation evenly among different processors or even machines by the well-known divide-and-conquer parallel paradigm [5, 22.

\subsection{MapReduce}

MapReduce [6] is a parallel programming technique, made popular by Google, used for processing large amounts of data. Such processing can be completed in a reasonable amount of time only by distributing the work to multiple machines in parallel. Each machine processes a small subset of the data. We will not discuss the details of MapReduce in this paper. 
List homomorphisms fit well with MapReduce, because their input list can be freely divided and distributed among machines. In fact, it has been shown recently that list homomorphisms can be efficiently implemented using MapReduce 19. Our approach builds on such an implementation which is orthogonal to our work. Therefore, if we can derive an efficient list homomorphism to solve a problem, we can solve the problem efficiently with MapReduce, enjoying its advantages such as automatic load-balancing, fault-tolerance, and scalability.

Some readers might feel that there is a mismatch between a typical MapReduce computation and computations in GTA style, because the size of the results generated by map in the former is often proportional to the size of the input data while the latter appears to have much larger intermediate results. This mismatch is a strength of our approach: based on a naively-designed GTA specification our calculation theorems can provide an efficient MapReduce implementation with intermediate results proportional to the size of the input, i.e., efficient list homomorphisms. Our approach makes MapReduce applicable to applications appearing not to match the MapReduce pattern. As a consequence, it allows programmers to implement MapReduce algorithms by providing an often simpler specification in GTA form.

\section{Running Example: The Knapsack Problem}

In this section we give a simple example of how to specify parallel algorithms in GTA form. We give a clear but inefficient specification of the knapsack problem following this structure and use it throughout Sections 4 and 5 to show how to transform such specifications into efficient parallel programs.

The knapsack problem is to fill a knapsack with items, each of certain value and weight, such that the total value of packed items is maximal while adhering to a weight restriction of the knapsack. For example, if the maximum total weight of our knapsack is $5 \mathrm{~kg}$ and there are three items $(¥ 2000,1 \mathrm{~kg}),(¥ 3000,3 \mathrm{~kg})$, and $(¥ 4000,3 \mathrm{~kg}$ ) then the best we can do is pick the selection ( $¥ 2000,1 \mathrm{~kg}$ ), ( $¥ 4000,3 \mathrm{~kg}$ ) with total value $¥ 6000$ and weight $4 \mathrm{~kg}$ because all selections with larger value exceed the weight restriction.

The function knapsack, which takes as input a list of value-weight pairs (both positive integers) and computes the maximum total value of a selection of items not heavier than a total weight $w$, can be written as a composition of three functions:

knapsack $=$ maxvalue $\circ$ filter $((\leqslant w) \circ$ weight $) \circ$ sublists

- The function sublists is the generator. From the given list of pairs it computes all possible selections of items, that is, all $2^{n}$ sublists if the input list has length $n$.

- The function filter $((\leqslant w) \circ$ weight $)$ is the test. It discards all generated sublists whose total weight exceeds $w$ and keeps the rest.

- The function maxvalue is the aggregator. From the remaining sublists adhering to the weight restriction it computes the maximum of all total values. 
The function sublists can be defined as follows:

$$
\begin{array}{ll}
\text { sublists }[] & =2[] \int \\
\text { sublists }[x] & =2[] \int \uplus 2[x] \int \\
\text { sublists }(x s+y s) & =\text { sublists } x s \times_{\#} \text { sublists ys }
\end{array}
$$

The result of sublists is a bag of lists which we denote using 2 and $\int$. The symbol $\uplus$ denotes bag union and $x_{\#}$ the lifting of list concatenation to bags, concatenating every list in one bag with every list in the other. The function sublists is a monoid homomorphism: $X_{H}$ is associative and 2[] $\int$ is its identity.

The function filter filters a bag according to the given predicate. We pass as predicate the composition of the function weight that adds all weights in a list and the function $(\leqslant w)$ that checks the weight restriction. Like sublists, weight is a monoid homomorphism:

$$
\begin{array}{ll}
\text { weight }[] & =0 \\
\text { weight }[(v, w)] & =w \\
\text { weight }(x s+y s) & =\text { weight } x s+\text { weight ys }
\end{array}
$$

Finally, maxvalue computes the maximum of summing up the values of each list in a bag using the maximum operator $\uparrow$.

$$
\begin{array}{ll}
\text { maxvalue } 2 \int_{\text {maxvalue }}\left[[] \int\right. & =-\infty \\
\text { maxvalue }\left\{[(v, w)] \int\right. & =v \\
\text { maxvalue }\left(b \uplus b^{\prime}\right) & =\text { maxvalue } b \uparrow \text { maxvalue } b^{\prime} \\
\text { maxvalue }\left(b \times_{+} b^{\prime}\right) & =\text { maxvalue } b+\text { maxvalue } b^{\prime}
\end{array}
$$

Regarding the last equation, remember that the lifted list concatenation $\times_{\mathbb{H}}$ appends each list in one bag with each in the other, and, therefore, the maximum total value of the concatenated lists is the sum of the maximum total values of the lists in each bag. This observation relies on distributivity of + over $\uparrow$, a property that we will revisit in the next section.

\section{Semiring Fusion}

In this section we show how to derive efficient parallel programs from specifications in generate-and-aggregate form:

aggregate o generate

This form is a simplified version of GTA form, missing the test. We define specific kinds of generators and aggregators that allow such specifications to be implemented efficiently and provide a theorem that shows how to calculate efficient parallel implementations. Such a calculation can turn an exponential-time specification into a linear-time implementation. 


\subsection{Semirings and Their Homomorphisms}

The specification for the function maxvalue in Section 3 shows that it is a monoid homomorphism with respect to two different monoids over the same set (bags of lists). We now consider an algebraic structure that relates two such monoids.

Definition 3 (Semiring). A triple $(S, \oplus, \otimes)$ is called a semiring if and only if $(S, \oplus)$ and $(S, \otimes)$ are monoids, and additionally $\oplus$ is commutative, $\otimes$ distributes over $\oplus$, and $\imath_{\oplus}$ is a zero of $\otimes$ :

$$
\begin{aligned}
& a \oplus b=b \oplus a \\
& a \otimes(b \oplus c)=(a \otimes b) \oplus(a \otimes c) \\
& (a \oplus b) \otimes c=(a \otimes c) \oplus(b \otimes c) \\
& \imath_{\oplus} \otimes a=\imath_{\oplus}=a \otimes \imath_{\oplus}
\end{aligned}
$$

We have already seen two semirings in Section 3 ,

$-\left(\mathbb{Z}_{-\infty}, \uparrow,+\right)$ is a semiring because both $\uparrow$ and + are associative, commutative and have identities $-\infty$ and 0 , respectively, where $\mathbb{Z}_{-\infty}=\mathbb{Z} \cup\{-\infty\}$. Moreover, + distributes over $\uparrow$ and $-\infty$ is a zero of + .

- ( $\left.[A] \int, \uplus, \mathrm{x}_{H}\right)$ is a semiring for every set $A$ because $\uplus$ is associative and commutative and $x_{+}$is associative. Moreover, $2 \int$ and 2[] $\int$ are the identities of $\uplus$ and $x_{\#}$, respectively. Interestingly, $x_{\#}$ distributes over $\uplus$ and, clearly, $\tau$ is a zero of $x_{H}$. Readers who verify distributivity of $x_{\#}$ will make crucial use of the ability to reorder bag elements.

Definition 4 (Semiring Homomorphism). Given two semirings $(S, \oplus, \otimes)$ and $\left(S^{\prime}, \oplus^{\prime}, \otimes^{\prime}\right)$, a function hom $: S \rightarrow S^{\prime}$ is a semiring homomorphism from $(S, \oplus, \otimes)$ to $\left(S^{\prime}, \oplus^{\prime}, \otimes^{\prime}\right)$ if and only if it is a monoid homomorphism from $(S, \oplus)$ to $\left(S^{\prime}, \oplus^{\prime}\right)$ and a monoid homomorphism from $(S, \otimes)$ to $\left(S^{\prime}, \otimes^{\prime}\right)$.

The maxvalue function presented in Section 3 is a semiring homomorphism from $\left(\imath\left[\mathbb{Z}_{-\infty} \times \mathbb{Z}_{-\infty}\right] \int, \uplus, \times_{+}\right)$to $\left(\mathbb{Z}_{-\infty}, \uparrow,+\right)$. It additionally satisfies the property maxvalue $2[(v, w)] \int=v$ which characterizes it uniquely because bags of lists over a set $A$ form the free semiring.

Lemma 1 (Free Semiring). Given a set $A$, a semiring $(S, \oplus, \otimes)$, and a function $f: A \rightarrow S$ there is exactly one semiring homomorphism $h: \eta[A] \int \rightarrow S$ from $\left(2[A] \int, \uplus, \mathrm{X}_{H}\right)$ to $(S, \oplus, \otimes)$ that satisfies $h \chi[x] \int=f x$.

The unique homomorphism can be thought of as applying $f$ to each list element, then accumulating the results in each list using the operator $\otimes$, and finally accumulating those results using the operator $\oplus$.

\subsection{Polymorphic Generators}

We now return to the generator sublists defined in Section 3. This function almost exclusively uses the semiring operations of the semiring $2[A] \int$ and their identities. The only exception is $2[x] \int$ constructed from an element $x \in A$. 
We can generalize sublists by parameterizing it with operations $\oplus$ and $\otimes$ of an arbitrary semiring (and their identities) as well as an embedding function that constructs semiring elements from elements of a (potentially) different type:

$$
\begin{array}{ll}
\text { sublist }_{\oplus, \otimes} f[] & =\imath_{\otimes} \\
\text { sublist }_{\oplus, \otimes} f[x] & =\imath_{\otimes} \oplus f x \\
\text { sublist }_{\oplus, \otimes} f(x s+\text { ys }) & =\text { sublists }_{\oplus, \otimes} f \text { xs } \otimes \text { sublist }_{\oplus, \otimes} f y s
\end{array}
$$

This function is called polymorphic over semirings because it can construct a result in an arbitrary semiring determined by the passed semiring operators and embedding function. It is a generalization of sublists because

$$
\text { sublists }=\text { sublists }_{\uplus, \chi_{+}}\left(\lambda x \rightarrow 2[x] \int\right)
$$

The anonymous function passed as argument constructs a singleton bag containing a singleton list with the argument $x$.

\section{Definition 5 (Polymorphic Semiring Generator). A function}

$$
\text { generate }_{\oplus, \otimes}:(A \rightarrow S) \rightarrow[A] \rightarrow S
$$

that is polymorphic over an arbitrary semiring $(S, \oplus, \otimes)$ is called a polymorphic semiring generator.

The function sublist $_{\oplus, \otimes}$ is a polymorphic semiring generator and, being a monoid homomorphism for any semiring, it can be executed in parallel. We could also pass the operations of the semiring $\mathbb{Z}_{-\infty}$ to compute a result in $\mathbb{Z}_{-\infty}$.

$$
\text { sublists } \uparrow,+(\lambda(v, w) \rightarrow v): 2\left[\mathbb{Z}_{-\infty} \times \mathbb{Z}_{-\infty}\right] \int \rightarrow \mathbb{Z}_{-\infty}
$$

What does this function compute? Theorem 1, which is a variant of short-cut fusion for semiring homomorphisms, casts light on this question.

Theorem 1 (Semiring Fusion). Given a set $A$, a semiring $(S, \oplus, \otimes)$, a semiring homomorphism aggregate from $\left(2[A] \int, \uplus, \mathrm{x}_{+}\right)$to $(S, \oplus, \otimes)$, and a polymorphic semiring generator generate, the following equation holds:

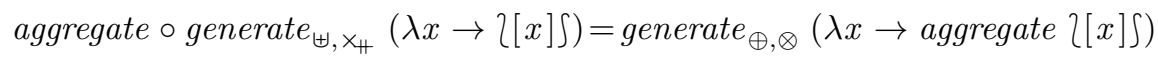

Proof. Free Theorem [1] [26].

We can use Theorem 1 to see what sublists $\uparrow,+(\lambda(v, w) \rightarrow v)$ computes.

$$
\begin{aligned}
& \text { maxvalue } \circ \text { sublists } \\
= & \text { maxvalue } \circ \text { sublist }_{\uplus, \mathrm{x}_{+}}\left(\lambda(v, w) \rightarrow 2[(v, w)] \int\right) \\
= & \text { sublists }_{\uparrow,+}\left(\lambda(v, w) \rightarrow \text { maxvalue } 2[(v, w)] \int\right) \\
= & \text { sublists }_{\uparrow,+}(\lambda(v, w) \rightarrow v)
\end{aligned}
$$

\footnotetext{
${ }^{1}$ The proof can be automated using an online tool: http://www-ps.iai.uni-bonn.de/ cgi-bin/free-theorems-webui.cgi
} 
This derivation shows that sublists $\uparrow,+(\lambda(v, w) \rightarrow v)$ computes the maximum of all total values of sublists of the input list, but - unlike the intuitive formulation at the beginning of the equation chain - efficiently. While the run time of maxvalue $\circ$ sublists is exponential in the length of the input list (because the result of sublists has exponential size), the run time of the derived version sublists $\uparrow,+(\lambda(v, w) \rightarrow v)$ is linear in the length of the input list (it adds up all positive values in the input).

Of course, this is of little use for solving the knapsack problem posed in Section 3 because the input list in this problem contains only positive values and maxvalue o sublists, thus, computes the total value of all available items.

For solving the knapsack problem, it is crucial to compute the maximum value only of those sublists of the input list which adhere to the weight restriction. We need to account for the test that implements this restriction which is the topic of the next section.

\section{$5 \quad$ Filter Embedding}

We cannot apply Theorem 1 to transform specifications of the form aggregate o test $\circ$ generate

because the intermediate test goes in the way of fusing the aggregator with the generator. We now show how specific instantiations of test allow to rewrite specifications like above into the form

$$
\text { postprocess o aggregate }{ }^{\prime} \circ \text { generate }
$$

where aggregate' is a semiring homomorphism derived from aggregate and test, and postprocess maps the result type of aggregate' to the result type of aggregate. This form then allows to fuse aggregate' with generate to derive an efficient implementation.

This transformation is possible if

$$
\text { test }=\text { filter }(\text { ok } \circ \text { hom })
$$

is a filter where the predicate is a composition of a monoid homomorphism hom $:[A] \rightarrow M$ into a finite monoid $M$ and a function $o k: M \rightarrow$ Bool that maps elements of $M$ to Booleans.

Before we describe the general theorem in Section 5.2, we develop the underlying ideas by deriving an efficient implementation from the knapsack specification. This development may seem to require some clever insights but users of our approach do not need to follow the same path when transforming their own specifications. We chose to present the ideas using a concrete example first, to make them seem less clever in the subsequent generalization. Others can simply apply our general theorem to their specifications rather than repeating our development for each specification on their own. We can even provide an API that supports specifications in GTA form and implements them as efficient parallel programs automatically. 


\subsection{Developing Intuitions by Example}

In Section 3 we have specified the knapsack function as follows:

$$
\text { knapsack }=\text { maxvalue } \circ \text { filter }((\leqslant w) \circ \text { weight }) \circ \text { sublists }
$$

This specification is almost of the form we require:

- maxvalue, the aggregator, is a semiring homomorphism and

- the predicate used for filtering is a composition of the monoid homomorphism weight and the function $(\leqslant w)$ that maps the result of weight into the Booleans.

However, the result type of weight is $\mathbb{N}$ which is an infinite monoid, not a finite one. We can remedy the situation by defining $M_{w}=\{0, \ldots, w+1\}$ and

$$
\begin{aligned}
& \text { weight }_{w}[] \quad=0 \\
& \text { weight }_{w}[n] \quad=(w+1) \downarrow n \\
& \text { weight }_{w}(m s+n s)=\text { weight }_{w} m s+{ }_{w} \text { weight }_{w} n s \\
& \quad \text { where } m+{ }_{w} n=(w+1) \downarrow(m+n)
\end{aligned}
$$

The operator $t_{w}$ implements addition but limits the result by computing the minimum with $w+1$ by using the minimum operator $\downarrow$. For non-negative arguments it is associative and 0 is its identity. Consequently, weight ${ }_{w}$ is a monoid homomorphism into the finite monoid $\left(M_{w},{ }_{w}\right)$ for all weight restrictions $w$.

To transform the function maxvalue $\circ$ filter $\left((\leqslant w) \circ\right.$ weight $\left._{w}\right)$ into the form postprocess $_{w} \circ$ maxvalue $_{w}$ we need to invent a semiring to use as result type of maxvalue $w_{w}$. The idea is to compute simultaneously for all weights in $M_{w}$ the maximum value of lists with exactly that weight. The function postprocess $_{w}$ then computes the maximum over all values associated to weights $\leqslant w$.

We use $w=5$ as an example, so semiring elements can be represented as 7 -tuples over $\mathbb{Z}_{-\infty}$. The function postprocess $s_{5}$ is defined as follows:

$$
\text { postprocess }_{5}\left(v_{0}, v_{1}, v_{2}, v_{3}, v_{4}, v_{5}, v_{6}\right)=v_{0} \uparrow v_{1} \uparrow v_{2} \uparrow v_{3} \uparrow v_{4} \uparrow v_{5}
$$

It computes the maximum of all values $v_{i}$ associated with weights $i \leqslant 5$. The entry $v_{6}$ for the weight 6 accumulates the maximum value corresponding to all weights $\geqslant 6$ because $t_{w}$ cuts off greater sums.

We now turn $\mathbb{Z}_{-\infty}^{7}$ into a semiring $\left(\mathbb{Z}_{-\infty}^{7}, \uparrow^{7},+^{7}\right)$. To compute the maximum value associated to each weight of two 7 -tuples, we use the underlying maximum operation on values.

$$
\begin{aligned}
& \left(v_{0}, v_{1}, v_{2}, v_{3}, v_{4}, v_{5}, v_{6}\right) \uparrow^{7}\left(v_{0}^{\prime}, v_{1}^{\prime}, v_{2}^{\prime}, v_{3}^{\prime}, v_{4}^{\prime}, v_{5}^{\prime}, v_{6}^{\prime}\right)= \\
& \quad\left(v_{0} \uparrow v_{0}^{\prime}, v_{1} \uparrow v_{1}^{\prime}, v_{2} \uparrow v_{2}^{\prime}, v_{3} \uparrow v_{3}^{\prime}, v_{4} \uparrow v_{4}^{\prime}, v_{5} \uparrow v_{5}^{\prime}, v_{6} \uparrow v_{6}^{\prime}\right)
\end{aligned}
$$

This operator clearly inherits associativity and commutativity from the underlying maximum operator and its identity is

$$
(-\infty,-\infty,-\infty,-\infty,-\infty,-\infty,-\infty)
$$


The operator $+^{7}$ is more interesting. From two 7 -tuples that associate maximum values to each weight in $M_{5}$ it computes another 7 -tuple that associates maximum values to the combined weights. For example, to find the maximum value associated to the weight 3 , it computes the maximum of all sums of values associated to weights that sum up to 3 (we omit the part for larger weights):

$$
\begin{aligned}
& \left(v_{0}, v_{1}, v_{2}, v_{3}, v_{4}, v_{5}, v_{6}\right)+{ }^{7}\left(v_{0}^{\prime}, v_{1}^{\prime}, v_{2}^{\prime}, v_{3}^{\prime}, v_{4}^{\prime}, v_{5}^{\prime}, v_{6}^{\prime}\right)= \\
& \quad\left(v_{0}+v_{0}^{\prime}\right. \\
& \quad,\left(v_{0}+v_{1}^{\prime}\right) \uparrow\left(v_{1}+v_{0}^{\prime}\right) \\
& \quad,\left(v_{0}+v_{2}^{\prime}\right) \uparrow\left(v_{1}+v_{1}^{\prime}\right) \uparrow\left(v_{2}+v_{0}^{\prime}\right) \\
& \quad,\left(v_{0}+v_{3}^{\prime}\right) \uparrow\left(v_{1}+v_{2}^{\prime}\right) \uparrow\left(v_{2}+v_{1}^{\prime}\right) \uparrow\left(v_{3}+v_{0}^{\prime}\right) \\
& \quad, \ldots)
\end{aligned}
$$

This operator is associative and its identity is

$$
(0,-\infty,-\infty,-\infty,-\infty,-\infty,-\infty)
$$

We now define maxvalue ${ }_{5}$ as the (cf. Lemma 1) semiring homomorphism that satisfies the following equation:

maxvalue $_{5} 2[(v, w)] \int=($ val 0 , val 1 , val 2 , val 3 , val 4 , val 5 , val 6$)$

where $v a l i=$ if $i \equiv(w \downarrow 6)$ then $v$ else $-\infty$

When applied to a singleton bag that contains a list with exactly one item,

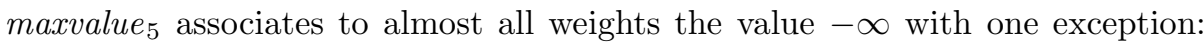
the value of the given item is associated to its weight (or to the weight 6 if it is heavier).

Our Main Theorem 3 below, now implies that for $w=5$

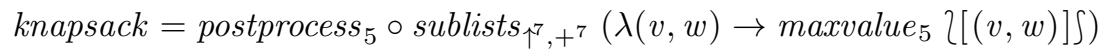

The run time of the transformed version of knapsack is $O\left(n w^{2}\right)$ if there are $n$ items and the weight restriction is $w$. As sublists $_{\uparrow^{7},+^{7}}$ is a monoid homomorphism we can execute it in parallel, say using $p$ processors, which leads to the run time $O\left(\left(\log p+\frac{n}{p}\right) w^{2}\right)$. This complexity resembles the run time of other parallel algorithms to solve the knapsack problem. The standard sequential algorithm has run time $O(n w)$.

Unlike existing algorithms to solve the knapsack problem, our approach can be generalized to other specifications in GTA form. The knapsack function is a special case well suited to highlight the ideas behind our approach, which we now generalize.

\subsection{The Generalized Theorem}

We now generalize the ideas of Section 5.1 to support

- arbitrary polymorphic semiring generators,

- arbitrary filters with homomorphic predicates, and

- arbitrary semiring homomorphisms as aggregators. 
In Section 5.1 we have used a semiring of 7-tuples storing maximum values corresponding to each weight in $M_{5}$. In general, if we have a finite monoid $M$ and a semiring $S$, then the set

$$
S^{M}=\left\{\left\{f_{m}\right\}_{m \in M} \mid f_{m} \in S\right\}
$$

of families of elements in $S$ indexed by $M$ is a semiring too. Indexed families are a generalization of tuples and we write $f_{m}$ for the element in $S$ indexed by the value $m \in M$ if $f \in S^{M}$ is an indexed family. We give definitions of indexed families by defining their value in $S$ for each $m \in M$.

Lemma 2 (Lifted Semiring). Given a finite monoid $(M, \odot)$ and a semiring $(S, \oplus, \otimes)$ the triple $\left(S^{M}, \oplus_{M}, \otimes_{M}\right)$ where

$$
\begin{aligned}
& \left(f \oplus_{M} f^{\prime}\right)_{m}=f_{m} \oplus f_{m}^{\prime} \\
& \left(f \otimes_{M} f^{\prime}\right)_{m}=\bigoplus_{\substack{k, l \in M \\
k \odot l=m}}\left(f_{k} \otimes f_{l}^{\prime}\right)
\end{aligned}
$$

is a semiring and

$$
\begin{aligned}
& \left(\imath_{\oplus_{M}}\right)_{m}=\imath_{\oplus} \\
& \left(\imath_{\otimes_{M}}\right)_{m}=\text { if } m \equiv \imath_{\odot} \text { then } \imath_{\otimes} \text { else } \imath_{\oplus}
\end{aligned}
$$

Proof. The monoid laws for $\oplus_{M}$ follow directly from those of $\oplus$. We leave the proof of the laws for $\otimes_{M}$ to interested readers.

The definition of $\oplus_{M}$ uses the underlying $\oplus$ operator just like the definition of $\uparrow^{7}$ in Section 5.1 uses $\uparrow$. The operator $\otimes_{M}$, like $+^{7}$, computes for each $m$ the maximum of all sums of values associated to weights that add up to $m$ if we instantiate $\odot$ and $\otimes$ with + and $\oplus$ with $\uparrow$. The identities also reflect their specific counterparts from Section 5.1

Intuitively, given a monoid homomorphism hom $:[A] \rightarrow M$, a semiring homomorphism aggregate: $\left\{[A] \int \rightarrow S\right.$, and a bag of lists $l s$, we can associate to $l s$ an indexed family $f^{l s} \in S^{M}$ that describes for each $m \in M$ the result of applying aggregate to a bag of exactly those lists $l \in l s$ that satisfy hom $l=m$ :

$$
f_{m}^{l s}=\operatorname{aggregate}(\text { filter }((m \equiv) \circ h o m) l s)
$$

Considering different instantiations for $l s$, we can observe the following identities:

$$
\begin{aligned}
f_{m}^{2 S} & =\imath_{\oplus} \\
f_{m}^{2[] S} & =\text { if } m \equiv \imath_{\odot} \text { then } \imath_{\otimes} \text { else } \imath_{\oplus} \\
f_{m}^{l s \uplus l s^{\prime}} & =f_{m}^{l s} \oplus f_{m}^{l s^{\prime}} \\
f_{m}^{l s x_{+} l s^{\prime}} & =\bigoplus_{\substack{k, l \in M \\
k \odot l=m}}\left(f_{k}^{l s} \otimes f_{l}^{l s^{\prime}}\right)
\end{aligned}
$$

They reflect the definitions of the semiring operations for $S^{M}$ and their identities. Because of these homomorphic equations for $f^{l s}$, we can compute $f^{l s}$ using a semiring homomorphism aggregate $_{M}$ that satisfies 


$$
\begin{aligned}
& \left(\text { aggregate }_{M} \chi[x] \int\right)_{m} \\
= & f_{m}^{\tau[x] S} \\
= & \text { aggregate }\left(\text { filter }((m \equiv) \circ \text { hom }) \chi[x] \int\right) \\
= & \text { if hom }[x] \equiv m \text { then aggregate }\left\{[x] \int \text { else } \imath_{\oplus}\right.
\end{aligned}
$$

According to Lemma 1 this semiring homomorphism is unique.

Definition 6 (Lifted Homomorphism). Given a set $A$, a finite monoid $(M, \odot)$, a monoid homomorphism hom from $([A],+)$ to $(M, \odot)$, a semiring $(S, \oplus, \otimes)$, and a semiring homomorphism aggregate from $\left(2[A] \int, \uplus, \mathrm{X}_{\#}\right)$ to $(S, \oplus, \otimes)$, the function

$$
\text { aggregate }_{M}: \imath[A] S \rightarrow S^{M}
$$

is the unique semiring homomorphism from $\left(\imath[A] \int, \uplus, \times_{\#}\right)$ to $\left(S^{M}, \oplus_{M}, \otimes_{M}\right)$ that satisfies

$$
\text { (aggregate } \left._{M} 2[x] \int\right)_{m}=\text { if hom }[x] \equiv m \text { then aggregate } 2[x] \int \text { else } \imath_{\oplus}
$$

The function aggregate $_{M}$ generalizes the function maxvalue $_{5}$ by using aggregate and $\imath_{\oplus}$ instead of maxvalue and $-\infty$.

Once we have computed $f^{l s}$, we can use a function $o k: M \rightarrow B o o l$ to combine all results $f_{m}^{l s}$ for $m \in M$ with ok $m=$ True to get the result of

$$
\underset{\substack{m \in M \\ m=\text { True }}}{\text { aggregate }}(\text { filter }(\text { ok } \circ \text { hom }) l s)=
$$

According to this equation, we can partition the bag of accepted lists according to elements of $M$ and aggregate them individually because aggregate is a semiring homomorphism. The postprocessor defined next combines such individual aggregations.

Definition 7 (Postprocessor). Given sets $M$ (finite) and $S$ and a function ok: $M \rightarrow$ Bool the function postprocess ${ }_{M}$ ok $: S^{M} \rightarrow S$ is defined as follows:

$$
\text { postprocess }_{M} \text { ok } f=\underset{\substack{m \in M \\ \text { ok } \\ m=\text { True }}}{\bigoplus} f_{m}
$$

It is clearly a generalization of postprocess ${ }_{5}$ which computes the maximum of all values associated to weights $\leqslant 5$.

We can now prove the theorem which constitutes the second half of our approach. It clarifies how to embed an arbitrary filter with a homomorphic predicate into an arbitrary semiring homomorphism.

Theorem 2 (Filter Embedding). Given a set $A$, a finite monoid $(M, \odot)$, a monoid homomorphism hom from $([A],+)$ to $(M, \odot)$, a semiring $(S, \oplus, \otimes)$, a semiring homomorphism aggregate from $\left(\left\{[A] \int, \uplus, \mathrm{X}_{\#}\right)\right.$ to $(S, \oplus, \otimes)$, and a function ok: $M \rightarrow$ Bool the following equation holds:

$$
\text { aggregate } \circ \text { filter }(\text { ok } \circ \text { hom })=\text { postprocess }_{M} \text { ok } \circ \text { aggregate }_{M}
$$


Proof. The following calculation combines previous observations and definitions to show the claimed identity.

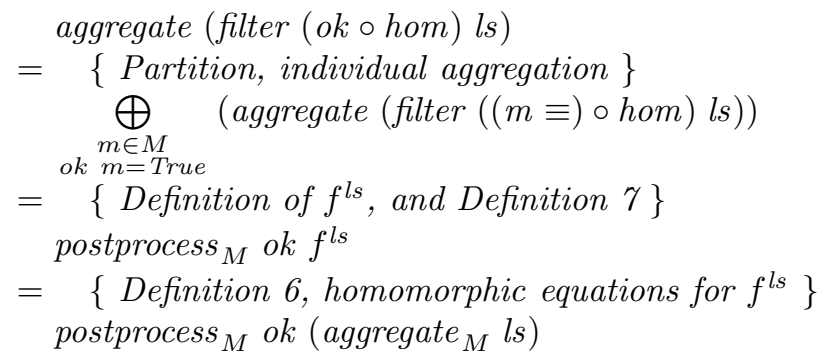

Our main result combines the theorems from Sections 4 and 5. It allows, under certain conditions, to transform specifications in GTA form into efficient parallel algorithms.

Main Theorem 3 (Filter-embedding Semiring Fusion). Given a set A, a finite monoid $(M, \odot)$, a monoid homomorphism hom from $([A],+)$ to $(M, \odot)$, a semiring $(S, \oplus, \otimes)$, a semiring homomorphism aggregate from $\left(2[A] \int, \uplus, \mathrm{X}_{\mathbb{H}}\right)$ to $(S, \oplus, \otimes)$, a function ok: $M \rightarrow$ Bool, and a polymorphic semiring generator generate, the following equation holds:

$$
\begin{aligned}
& \text { aggregate } \circ \text { filter }(\text { ok } \circ \text { hom }) \circ \text { generate }_{\uplus, x_{+}}\left(\lambda x \rightarrow 2[x] \int\right) \\
& =\text { postprocess }_{M} \text { ok } \circ \text { generate }_{\oplus_{M}, \otimes_{M}}\left(\lambda x \rightarrow \text { aggregate }_{M}\left[[x] \int\right)\right.
\end{aligned}
$$

Proof. Combining Theorems 1 and 2,

Filter-embedding Semiring Fusion is not restricted to parallel algorithms. It can be used to calculate efficient programs from specifications that use arbitrary polymorphic semiring generators.

It is worth noting that it is possible to remove the finiteness requirement for monoids and define a lifted semiring of finite mappings of unbounded and unknown size. We require the finiteness only in order to be able to describe the complexity of the resulting parallel algorithms more accurately.

If the generator happens to be a list homomorphism, like sublists, then associativity of list concatenation allows the resulting program to be executed in parallel by distributing the input list evenly among available processors. The complexity of a derived program using sublists as generator is linear in the size of the input list and quadratic in the size of the range $M$ of the homomorphic predicate because the semiring multiplication of the lifted semiring $S^{M}$, which is used to combine all list elements, can be implemented by ranging over $M \times M$.

\section{A More Complex Application}

In this section we describe how to use our framework to derive an efficient parallel implementation for a practical problem in statistics. We further demonstrate how to extend the derived basic program incrementally. 


\subsection{Finding a Most Likely Sequence of Hidden States}

We now revisit the statistics problem mentioned in Section [1 which is to find a sequence of hidden states of a probabilistic model that most likely causes a sequence of observed events. For example, for speech recognition, the acoustic signal could be the sequence of observed events, and a string of text the sequence of hidden states.

Given a sequence $x=\left(x_{1}, \ldots, x_{n}\right)$ of observed events, a set $S$ of states in a hidden Markov model, probabilities $P_{\text {yield }}\left(x_{i} \mid z_{j}\right)$ of events $x_{i}$ being caused by states $z_{j} \in S$, and probabilities $P_{\text {trans }}\left(z_{i} \mid z_{j}\right)$ of states $z_{i}$ appearing immediately after states $z_{j}$, the objective is to find a sequence $z=\left(z_{0}, \ldots, z_{n}\right)$ of hidden states that is most likely to cause the sequence $x$ of events such that every $z_{i}$ causes $x_{i}$ for $i>0$ and $z_{0}$ is an initial state. This problem can be formalized by the following expression.

$$
\underset{z \in S^{n+1}}{\arg \max }\left(\prod_{i=1}^{n} P_{\text {yield }}\left(x_{i} \mid z_{i}\right) P_{\text {trans }}\left(z_{i} \mid z_{i-1}\right)\right)
$$

To derive an efficient parallel algorithm to solve this problem, we transform this expression to fit in our framework.

To eliminate the index $i-1$, we let the expression range over pairs of hidden states in $S \times S$ and introduce a predicate trans to restrict the considered lists of state pairs. Intuitively, trans $y$ is True if and only if the given sequence $y$ of state pairs describes consecutive transitions

$$
\left(\left(z_{0}, z_{1}\right),\left(z_{1}, z_{2}\right), \ldots,\left(z_{n-2}, z_{n-1}\right),\left(z_{n-1}, z_{n}\right)\right)
$$

and False otherwise. Introducing the function

$$
\operatorname{prob}(x,(s, t))=P_{\text {yield }}(x \mid t) P_{\text {trans }}(t \mid s)
$$

the expression above can be transformed into the following equivalent expression.

$$
\underset{\substack{y \in(S \times S)^{n} \\ \text { trans } y=\text { True }}}{\arg \max }\left(\prod_{i=1}^{n} \operatorname{prob}\left(x_{i}, y_{i}\right)\right)
$$

In a first step, we specify only the maximum probability in GTA form. We show how to compute a state sequence corresponding to this probability by using a different aggregator later.

Representing sequences of states and events as lists, we can write the transformed specification as follows.

$$
\begin{aligned}
\text { maxLikeliness }= & \text { maxprob } \circ \\
& \text { filter }(\text { trans } \circ \text { map }(\lambda(x,(s, t)) \rightarrow(s, t))) \circ \\
& \text { assignTrans } s_{\uplus, \chi_{\#}}\left(\lambda x \rightarrow 2[x] \int\right)
\end{aligned}
$$

The polymorphic semiring generator assign Trans ${ }_{\oplus, \otimes}$ is defined as the unique monoid homomorphism from $([X],+)$ to the multiplicative monoid $(T, \otimes)$ of an arbitrary semiring $(T, \oplus, \otimes)$ that satisfies 
assignTrans $_{\oplus, \otimes} f[x]=$ reduce $_{\oplus}[f(x,(s, t)) \mid s \leftarrow S, t \leftarrow S]$

Here, reduce $_{\oplus}$ is a monoid homomorphism from $([T],+)$ to $(T, \oplus)$ that satisfies reduce $_{\oplus}[x]=x$. Intuitively, assignTrans $s_{\uplus, \chi_{+}}\left(\lambda x \rightarrow 2[x] \int\right)$ produces a bag of event sequences where all possible combinations of state transitions are attached.

The predicate trans is defined as not $\circ(\square \equiv) \circ$ reduce $\diamond$ where reduce $e_{\diamond}$ is a monoid homomorphism from $([S \times S],+)$ to the finite monoid $\left((S \times S)_{\square}, \diamond\right)$ and $(S \times S)_{\square}$ is $(S \times S) \cup\left\{\imath_{\diamond}, \square\right\}$. Here, $\square$ is a zero of $\diamond$ and

$$
(s, t) \diamond(u, v)=\text { if } t \equiv u \text { then }(s, v) \text { else } \square
$$

Intuitively, reduce $\diamond$ returns the boundaries of a given sequence of state transitions if they are consecutive ( $\iota_{\diamond}$ if the sequence is empty) and $\square$ otherwise.

The aggregator maxprob is the unique semiring homomorphism from $(2[X \times$ $\left.(S \times S)] \int, \uplus, \times_{\mathbb{H}}\right)$ to $([0,1], \uparrow, * \sqrt{2}$ that satisfies

$$
\text { maxprob } 2[(x,(s, t))] \int=\operatorname{prob}(x,(s, t))
$$

Intuitively, it computes all total probabilities of state sequences causing the observed event sequence by multiplying the individual probabilities given by prob and then computes the maximum of all total probabilities.

The range of reduce $\diamond$ has size $|S|^{2}+2$, thus, applying Theorem 3 to the specification of maxLikeliness yields an implementation with the total cost $O\left(n|S|^{4}\right)$ if $n$ denotes the length of an input event sequence. As assignTrans is a monoid homomorphism we can execute it in parallel, say using $p$ processors, which leads to the run time $O\left(\left(\log p+\frac{n}{p}\right)|S|^{4}\right)$. For a given probabilistic model, where $S$ is fixed, the result is a linear-time parallel algorithm. This is in contrast to the specification which would generate an intermediate result of size $|S|^{2 n}$. Interestingly, the derived program is equivalent to a program obtained by parallelizing the Viterbi algorithm [12,13] using matrix multiplication over a semiring [21.

Computing Sequences of States. We can compute both the maximum probability and the corresponding state sequences using an alternative aggregator maxprobSeq which can replace maxprob above and is characterized by

$$
\text { maxprobSeq } 2[(x,(s, t))] \int=\left(\operatorname{prob}(x,(s, t)), 2[t] \int\right)
$$

The result is an element in the semiring $\left([0,1] \times 2[S] \int, \uparrow^{\prime}, *^{\prime}\right)$ where the identities of $\uparrow^{\prime}$ and $*^{\prime}$ are $\left(0,2 \int\right)$ and $\left(1,2[] \int\right)$, respectively, and the semiring operations are defined as follows:

$$
\begin{aligned}
& (a, x) \uparrow^{\prime}(b, y)=\text { if } a>b \text { then }(a, x) \text { else if } a<b \text { then }(b, y) \text { else }(a, x \uplus y) \\
& (a, x) *^{\prime}(b, y)=\left(a * b, x \times_{\mathbb{H}} y\right)
\end{aligned}
$$

The bag in the second component of the result contains all most likely sequences. In practice, we may use non-deterministic choice to compute one of them, though operators with non-deterministic choice do not satisfy the semiring laws, so the specification and the implementation might pick different results.

${ }^{2}$ To avoid confusion, note that $[0,1]$ is the unit interval, that is, the set of all real numbers $x$ such that $0 \leq x \leq 1$, not the list of the two elements. 


\subsection{Incremental Refinement}

By using Theorem 2 multiple times, it is possible to implement specifications with multiple filters, not only one.

For example, we can compute the most likely sequence of hidden states satisfying certain conditions, such as "state $s$ is used exactly five times," or "state $t$ does not appear anywhere after state $s . "$ Our framework guarantees an efficient implementation also for these restricted problems if the conditions can be defined by a homomorphic predicate.

For the first condition we use the monoid homomorphism count $_{w} p$ into $\left(M_{w},{ }_{w}\right)$ characterized by count ${ }_{w} p[x]=$ if $p x$ then 1 else 0 . It computes the number of list elements that satisfy the given predicate $p$. Based on count $_{w}$ we can define the predicate fixedTimes which only allows sequences of states that contain a given state $s$ exactly $w$ times:

$$
\text { fixedTimes } s w=(w \equiv) \circ \text { count }_{w}(\lambda(x,(t, u)) \rightarrow s \equiv u)
$$

To check the second condition whether a state $t$ occurs anywhere after a state $s$ we can define a monoid homomorphism after $s t$ into $\left((B o o l \times B o o l)_{\square}, \star\right)$ that returns a pair of Booleans that indicate whether the argument list contains the states $s$ and $t$, or $\square$ if $t$ occurs anywhere after $s{ }^{3}$ Here, after is characterized by

$$
\text { after } s t[(x,(u, v))]=(s \equiv v, t \equiv v),
$$

is a zero of $\star$ and $\left(s_{1}, t_{1}\right) \star\left(s_{2}, t_{2}\right)=$ if $s_{1} \wedge t_{2}$ then $\square$ else $\left(s_{1}\left|s_{2}, t_{1}\right| t_{2}\right)$. Based on after we can express a test which only allows sequences of states that do not contain a given state $t$ after $s$ as not $\circ(\square \equiv) \circ$ after $s t$.

Since both homomorphisms have finite ranges, we can get linear-time parallel algorithms for the restricted problems. We can even combine both predicates or add similar conditions such as "state $s$ is used more than $k$ times," or "state $s$ is used at most $k$ times" and still get an efficient parallel implementation.

In general, the most difficult task for programmers specifying GTA algorithms is the design of predicates for filtering, while basic generators and aggregators can be reused for many problems. To guarantee the efficiency of programs derived by our calculation theorems, a user has to design a predicate based on a finite monoid. One approach to design such a predicate is to use a regular expression or monadic second order logic expression 25], relying on the fact that a finite monoid can be derived from a finite automaton. For example, an additional condition "we cannot choose items $\mathrm{K}$ and $\mathrm{J}$ at the same time" to the knapsack problem can be specified by a regular expression (.* K.* J.*|.* J.* K.*) composed with the negation function not.

\section{Related Work}

The research on parallelization via derivation of list homomorphisms has gained great interest [5, 11,22. The main approaches include the third homomorphism

$\overline{{ }^{3}(\text { Bool } \times \text { Bool })} \square=($ Bool $\times$ Bool $) \cup\{\square\}$ and $\imath_{\star}=($ False, False $)$. 
theorem based method [10, 20], function composition based method [4, 7, 14, and matrix multiplication based method 21. Our work is a continued effort in this direction, giving a new approach based on semiring homomorphisms, which is in sharp contrast to the existing work based on monoid homomorphisms. By introducing bags of lists as well as semirings and the GTA form, our method eases defining effectively-parallelizable specifications for practical problems such as the knapsack problem, the discussed statistical problems, and querying problems, because the GTA form with bag of lists is a natural form of specifications for these combinatorial problems. Basically, specifications of these problems are too complex to be handled by the mentioned previous approaches. The previous work cannot directly help users to solve these problems, because it requires users to make parallelizable sequential specifications that are almost equivalent to the efficient programs our proposed method derives. However, previous approaches are still useful to build a parallelizable GTA specification which requires its components (generators and predicates) to be parallel programs.

There has been a lot of work about using MapReduce to parallelize various kinds of problems [17. Some formal work has been devoted to the study of a computation model of MapReduce (compared to the PRAM model of computation) [16. However, little work has been done on systematic construction of MapReduce programs. We tackle this problem via semiring homomorphisms.

Our shortcut fusion theorem for semiring fusion is much related to the known shortcut deforestation [8, 23, which is based on a free theorem [26] and is practically useful for optimization of sequential programs. Different from the traditional shortcut deforestation focusing on the data constructors of the intermediate data structure that are passed from one function to another, our shortcut fusion focuses on the semiring operations in the intermediate data structure. It is this semiring structure that allows for flexible rearrangement of computation for efficient parallel execution.

Goodman 9] extended the CYK parsing algorithm by substituting various semirings for the Boolean semiring, so that one can reuse the algorithm for various computations such as counting the number of parsings, computing the probability of generating the given string, and finding the best $k$-parsing. We can reuse his semirings in our GTA form for computing similar variations.

\section{Conclusion}

We propose a calculation-based framework for the systematic development of efficient MapReduce programs in the form of GTA algorithms. The core of the framework consists of two calculation theorems for semiring fusion and filter embedding. Semiring fusion connects a specification in GTA form and an efficient implementation by a free theorem, while filter embedding transforms the composition of a semiring homomorphism and a test into another semiring homomorphism which enables incremental development of parallel algorithms. Our approach allows to develop efficient parallel algorithms by combining simpler homomorphisms (for generation, testing, and aggregation) into more complex ones, 
which is easier than defining the efficient parallel algorithms directly. In contrast to existing approaches, our theorems allow to modify an efficient algorithm by adding homomorphic filters in the "naive world" which is easier than modifying it in the "efficient world". Our new framework is not only theoretically interesting, but also practically significant in solving nontrivial problems.

For example, we have shown how to derive an efficient parallel implementation of a known statistics problem and found that it is equivalent to an existing algorithm for the same problem. This result shows that our approach generalizes existing techniques and provides a common framework to express them. We expect that our approach can be applied to typical "big-data" problems, like finding patterns in historical financial data, and plan to investigate such applications as future work.

Moreover, we plan to implement the developed programming theory as a domain specific language or a library, for example upon Hadoop [27, so that typical MapReduce problems can be tackled using our GTA approach. Our theorems can be easily mechanized because of their simple calculational form.

\section{References}

1. Bird, R.: An introduction to the theory of lists. In: Broy, M. (ed.) Logic of Programming and Calculi of Discrete Design, pp. 5-42. Springer, Heidelberg (1987)

2. Bird, R.: Introduction to Functional Programming using Haskell. Prentice Hall (1998)

3. Bird, R., de Moor, O.: Algebras of Programming. Prentice Hall (1996)

4. Chin, W.N., Khoo, S.C., Hu, Z., Takeichi, M.: Deriving Parallel Codes via Invariants. In: SAS 2000. LNCS, vol. 1824, pp. 75-94. Springer, Heidelberg (2000)

5. Cole, M.: Parallel programming with list homomorphisms. Parallel Processing Letters 5(2), 191-203 (1995)

6. Dean, J., Ghemawat, S.: Mapreduce: simplified data processing on large clusters. Communications of the ACM 51, 107-113 (2008)

7. Fisher, A.L., Ghuloum, A.M.: Parallelizing complex scans and reductions. In: Proceedings of the ACM SIGPLAN 1994 Conference on Programming Language Design and Implementation (PLDI 1994), pp. 135-146. ACM (1994)

8. Gill, A., Launchbury, J., Peyton Jones, S.L.: A short cut to deforestation. In: Conference on Functional Programming Languages and Computer Architecture, pp. 223-232 (1993)

9. Goodman, J.: Semiring parsing. Computational Linguistics 25, 573-605 (1999)

10. Gorlatch, S.: Systematic Efficient Parallelization of Scan and Other List Homomorphisms. In: Fraigniaud, P., Mignotte, A., Robert, Y., Bougé, L. (eds.) Euro-Par 1996. LNCS, vol. 1124, pp. 401-408. Springer, Heidelberg (1996)

11. Grant-Duff, Z., Harrison, P.: Parallelism via homomorphism. Parallel Processing Letters 6(2), 279-295 (1996)

12. He, Y.: Extended viterbi algorithm for second order hidden markov process. In: 9th International Conference on Pattern Recognition, vol. 2, pp. 718-720. IEEE Press (1988)

13. Ho, T.J., Chen, B.S.: Novel extended viterbi-based multiple-model algorithms for state estimation of discrete-time systems with markov jump parameters. IEEE Transactions on Signal Processing 54(2), 393-404 (2006) 
14. Hu, Z., Takeichi, M., Chin, W.N.: Parallelization in calculational forms. In: 25th ACM Symposium on Principles of Programming Languages (POPL 1998), pp. 316-328. ACM Press, San Diego (1998)

15. Hu, Z., Yokoyama, T., Takeichi, M.: Program Optimizations and Transformations in Calculation Form. In: Lämmel, R., Saraiva, J., Visser, J. (eds.) GTTSE 2005. LNCS, vol. 4143, pp. 144-168. Springer, Heidelberg (2006)

16. Karloff, H., Suri, S., Vassilvitskii, S.: A model of computation for mapreduce. In: Proceedings of the Twenty-First Annual ACM-SIAM Symposium on Discrete Algorithms, SODA 2010, pp. 938-948. SIAM (2010)

17. Lin, J., Dyer, C.: Data-Intensive Text Processing with MapReduce. Morgan and Claypool Publishers (2010)

18. List, M.A.P. (2011), http://www.mendeley.com/groups/1058401/mapreduceapplications/papers/

19. Liu, Y., Hu, Z., Matsuzaki, K.: Towards Systematic Parallel Programming over MapReduce. In: Jeannot, E., Namyst, R., Roman, J. (eds.) Euro-Par 2011, Part II. LNCS, vol. 6853, pp. 39-50. Springer, Heidelberg (2011)

20. Morita, K., Morihata, A., Matsuzaki, K., Hu, Z., Takeichi, M.: Automatic inversion generates divide-and-conquer parallel programs. In: ACM SIGPLAN 2007 Conference on Programming Language Design and Implementation (PLDI 2007), pp. 146-155. ACM Press (2007)

21. Sato, S., Iwasaki, H.: Automatic parallelization via matrix multiplication. In: Proceedings of the 32nd ACM SIGPLAN Conference on Programming Language Design and Implementation (PLDI 2011), pp. 470-479. ACM (2011)

22. Skillicorn, D.B.: The Bird-Meertens Formalism as a Parallel Model. In: NATO ARW "Software for Parallel Computation" (1992)

23. Takano, A., Meijer, E.: Shortcut deforestation in calculational form. In: Proc. Conference on Functional Programming Languages and Computer Architecture, pp. 306-313. La Jolla, California (1995)

24. Takano, A., Hu, Z., Takeichi, M.: Program transformation in calculational form. ACM Computing Surveys 30(3) (1998)

25. Thomas, W.: Automata on infinite objects. In: Handbook of Theoretical Computer Science, Formal Models and Sematics, vol. B, pp. 133-192. Elsevier and MIT Press (1990)

26. Wadler, P.: Theorems for free! In: Proceedings of the Fourth International Conference on Functional Programming Languages and Computer Architecture, FPCA 1989, pp. 347-359. ACM (1989)

27. White, T.: Hadoop: The Definitive Guide. O'Reilly Media (2009) 